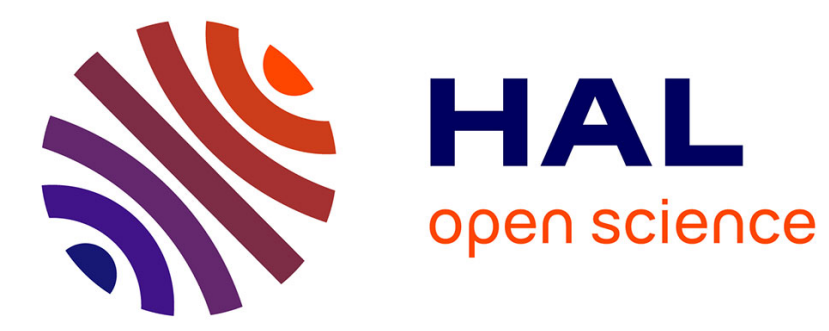

\title{
Modélisation par méthode variationnelle d'un actionneur piézoélectrique à rotation de mode. Approche analytique et numérique
}

\author{
Marc Budinger, Jean-François Rouchon, Bertrand Nogarède
}

\section{To cite this version:}

Marc Budinger, Jean-François Rouchon, Bertrand Nogarède. Modélisation par méthode variationnelle d'un actionneur piézoélectrique à rotation de mode. Approche analytique et numérique. European Journal of Electrical Engineering, 2004, 7 (1-2), pp.223-251. 10.3166/rige.7.223-251 . hal-01861768

\author{
HAL Id: hal-01861768 \\ https://hal.science/hal-01861768
}

Submitted on 27 Aug 2018

HAL is a multi-disciplinary open access archive for the deposit and dissemination of scientific research documents, whether they are published or not. The documents may come from teaching and research institutions in France or abroad, or from public or private research centers.
L'archive ouverte pluridisciplinaire $\mathbf{H A L}$, est destinée au dépôt et à la diffusion de documents scientifiques de niveau recherche, publiés ou non, émanant des établissements d'enseignement et de recherche français ou étrangers, des laboratoires publics ou privés. 


\title{
Modélisation par méthode variationnelle d'un actionneur piézoélectrique à rotation de mode Approche analytique et numérique
}

\author{
M. Budinger, J-F. Rouchon, B. Nogarede \\ Laboratoire d'Electrotechnique et d'Electronique Industrielle \\ (LEEI), Equipe Machines et Mécanismes Electroactifs $\left(\mathbf{E M}^{2}\right)$ \\ 2,rue Camichel \\ 31071 Toulouse \\ France \\ nom@leei.enseeiht.fr
}

RÉSUMÉ. L'article a pour but le calcul des caractéristiques électromécaniques d'un actionneur piézoélectrique à rotation de mode (moteur à onde progressive). L'utilisation d'une méthode énergétique par Lagrangien permet de déterminer les paramètres caractéristiques de la conversion électromécanique de l'énergie. Cette étape peut être réalisée par des calculs purement analytiques à l'aide d'une fonction d'approximation de la déformée au sein du transducteur ou à l'aide d'un logiciel par éléments finis (ANSYS). Une exemple simple de transducteur Langevin illustre dans un premier temps cette approche. Le stator du moteur à rotation de mode est ensuite traité. La prise en compte de la conversion mécano-mécanique de l'énergie entre le stator et le rotor du moteur est quant à elle traitée de manière purement analytique. L'ensemble permet d'accéder à la courbe couple vitesse, la fréquence de résonance, la tension d'alimentation du moteur. Les méthodes utilisées peuvent s'adapter à d'autre types d'actionneurs piézoélectriques.

ABSTRACT.

MOTS-CLÉS : actionneurs piézoélectrique, modélisation, lagrangien, calcul analytique, calcul numérique (FEM).

KEYWORDS: piezoelectric actuators, modeling, lagrangien, analytical calcul, numerical calcul (FEM).

RIGE - proposition d'article 


\section{Introduction}

Les actionneurs piézo-électriques présentent des avantages désormais bien connus face à d'autres technologies pour tout système mécanique embarqué qui requiert un fort couple massique, un système freiné à l'arrêt, et un confort d'utilisation accru [SAS][UEH]. Parmi ces actionneurs, le moteur à rotation de mode, de forme cylindrique et à onde progressive, présente des caractéristiques intéressantes en terme de conversion d'énergie : bonne utilisation des céramiques au niveau de la conversion électromécanique d'énergie électrique en vibrations mécaniques, bonne transmission des efforts vibratoires par un contact permanent entre stator et rotor pour la conversion mécano-mécanique. La modélisation d'actionneur à onde progressive de forme annulaire a déjà été entrepris tant au niveau du contact [MIN 96][ROU 95] ou de la modélisation variationelle de la conversion électromécanique [HAG 95][GIR 98]. L'article s'attachera à utiliser ces méthodes de manière simplifiée pour modéliser le moteur à rotation de mode et montrera comment calculer les paramètres du moteur de manière analytique ou numérique.

\section{Modélisation par approche variationnelle}

Cette partie rappelle brièvement comment le calcul d'efforts électromécaniques par variation d'énergie électrique stockée doit être étendu pour s'adapter aux milieux piézoélectriques. La référence [NOG] introduit de manière plus détaillée l'ensemble de ces concepts. La méthode exposée est illustrée sur un exemple simple de transducteur Langevin.

\subsection{Lagrangien d'un transducteur piézoélectrique}

Un système électromécanique simple, sans couplage fort entre énergie électrique et énergie mécanique (pas d'énergie mécanique stockée) et possédant une phase alimentée par une tension $v$ et générant un effort $f$ selon une déformée $x$, transforme l'énergie électrique apportée par l'alimentation $d W_{a \lim }=v \cdot d q_{c}$ en une variation d'énergie électrique stockée $\delta W_{E}^{q}\left(q_{c}, x\right)$ et en un travail mécanique $d W_{\text {meca }}=f \cdot d x$ :

$$
v d q_{c}=\delta W_{E}^{q}\left(q_{c}, x\right)+f d x
$$

Cette équation permettrait d'exprimer $f$ en supposant la variation de charge nulle. Cette hypothèse est rarement vérifiable en pratique, car on dispose généralement d'une source de tension. On préfère donc introduire la coénergie électrique $W_{E}{ }^{u}(v, x)$ qui vérifie : 


$$
W_{E}^{q}(q, x)+W_{E}^{v}(v, x)=v q_{c}
$$

Le nouveau bilan d'énergie comportant cette coénergie permet alors d'exprimer l'effort $f$ en supposant la tension d'alimentation constante :

$$
f=\left(\frac{\partial W_{E}^{v}(v, x)}{\partial x}\right)_{v=C t e}
$$

De même en supposant le déplacement constant, on exprime alors la charge $q_{c}$ par:

$$
q_{c}=\left(\frac{\partial W_{E}^{v}(v, x)}{\partial v}\right)_{x=C t e}
$$

Dans le cas de système linéaire, la coénergie électrique s'exprime par :

$$
W_{E}^{v}(U, x)=\int_{0}^{v} q_{c} d v=\iint D d E d \Omega=\frac{1}{2} \int E D d \Omega
$$

Pour un milieu piézoélectrique, à couplage fort entre énergie électrique et mécanique, cette approche est insuffisante : à une variation d'énergie électrique emmagasinée correspond toujours une variation d'énergie élastique de déformation mécanique. De plus pour les actionneurs piézoélectriques excités en dynamique, à la résonance par exemple, l'énergie mécanique peut être également stockée sous forme d'énergie cinétique. Le Lagrangien permet de prendre en compte ces termes énergétiques complémentaires.

Pour un milieu purement mécanique, dont la description cinématique peut se réduire à un ensemble de coordonnées généralisées $q_{j}$, le Lagrangien $L$ s'écrit sous la forme :

$$
L=T-V-V_{e}
$$

Avec :

- $T$, l'énergie cinétique du milieu en mouvement : $T=\int \frac{1}{2} \rho \dot{u}^{2} d \Omega$;

- $\quad V$, le potentiel des forces conservatives comme celles des déformations élastiques : $V=\int \frac{1}{2} T S d \Omega$;

- $V_{e}$, le pseudo-potentiel des forces généralisées extérieures $f_{j}{ }^{e}$ agissant sur les degrés de libertés $q_{j}: V_{e}=-f_{j}^{e} q_{j}$. 
Ce Lagrangien vérifie pour chacune de ses coordonnées généralisées :

$$
\frac{d}{d t} \frac{\partial L}{\partial \dot{q}_{j}}-\frac{\partial L}{\partial q_{j}}=0
$$

Pour un milieu piézoélectrique, aux forces de déformation élastique $f_{j}=-\partial V / \partial q_{j}$ s'ajoute les efforts d'origine électromécanique. Le potentiel $-W_{E}{ }^{v}$ de la coénergie électrique vient compléter le terme $V$. Au pseudo-potentiel des forces mécaniques extérieures s'ajoute le terme $v q_{c}$ qui permet de tenir compte de l'équation électrique [4.]. Le Lagrangien d'un transducteur piézoélectrique prend donc finalement pour expression :

$$
L\left(\dot{q}_{j}, q_{j}, v\right)=\frac{1}{2} \int\left(\rho \dot{u}^{2}-T S+E D\right) d \Omega+\sum f_{j}^{e} q_{j}-v q_{c}
$$

Ce Lagrangien peut être calculé à l'aide d'une approximation du champ de déplacement $u$. Cette approximation peut être réalisée à l'aide d'une seule fonction (méthode de Rayleigh) ou d'une somme de fonctions (méthode de Rayleigh-Ritz). La suite de l'article utilisera la première méthode, toutes les déformations au sein du transducteur seront donc fonction d'un seul paramètre géométrique qui servira de coordonnées mécanique généralisée. L'intégration des différentes composantes du Lagrangien donnera une fonction de la forme :

$$
L(\dot{q}, q, v)=\frac{1}{2} M \dot{q}^{2}-\frac{1}{2} K q^{2}+\frac{1}{2} C_{0} v^{2}+N v q+f q-v q_{c}
$$

La différentiation de ce Lagrangien selon les coordonnées $q$ et $v$ permettra d'obtenir les équations caractéristiques du transducteurs :

- Equation mécanique : $M \ddot{q}+K q=N v+f$, avec la masse $M$, la raideur $K$ et le facteur d'effort $N$;

- Equation électrique : $q_{c}=N q+C_{0} v$, avec la capacité $C_{0}$.

\subsection{Modélisation d'un transducteur Langevin}

Un transducteur piézoélectrique est généralement constitué d'une céramique accolée à une structure métallique en contact avec la charge mécanique. Une modélisation autour de la résonance d'un transducteur simplifié de cette forme permet d'obtenir les grandeurs intéressantes en terme de dimensionnement : masse, raideur modale et facteur d'effort. Le transducteur étudié est du type Langevin symétrique. Il est réalisé à l'aide de contre-masses encadrant des céramiques piézo-électriques . Les propriétés mécaniques (densité et élasticité) des différents matériaux sont supposées similaires. 


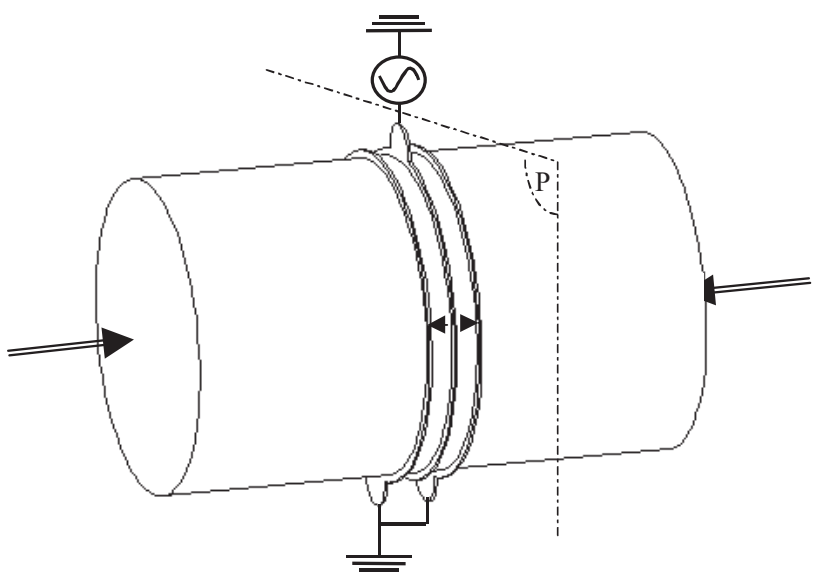

Figure 3. Géométrie du transducteur Langevin

Calcul analytique des paramètres

Ce transducteur de type Langevin excite par ses céramiques piézo-électriques l'ensemble de la structure élastique selon un mode longitudinal. Pour des fréquences proches de la résonance, les déformées au sein du transducteur peuvent se calculer en établissant l'équation locale de mouvement d'une barre en extension [Géradin]. Un élément, en $x$, de longueur $d x$ du transducteur qui est caractérisé par une déformée $u(x)$ et une vitesse $\dot{u}(x)$ et vérifie :

$$
\rho \ddot{u}-c \frac{\partial^{2} u}{\partial x^{2}}=0
$$

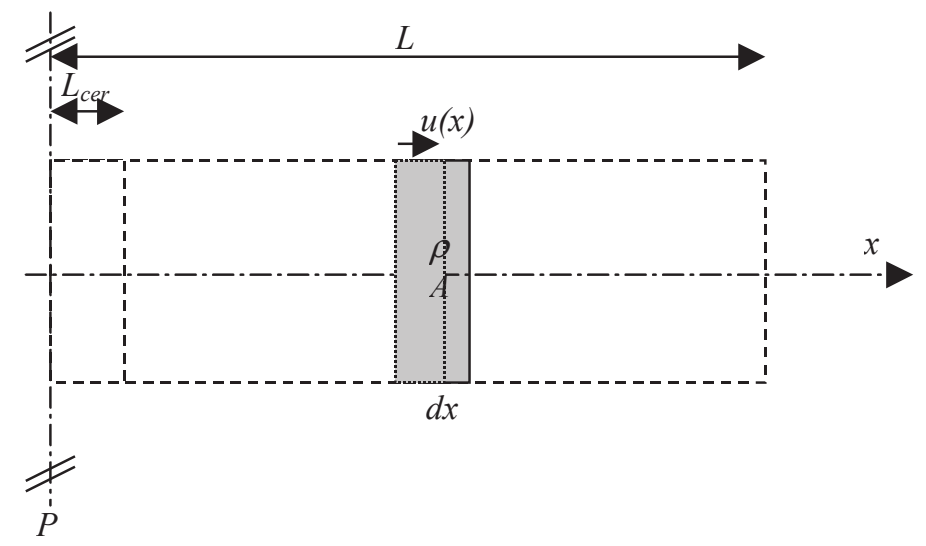

Figure 1. Elément de barre en extension 
Pour la configuration symétrique considérée, cette équation admet une solution du type :

$$
u(x, t)=\sin \left(\frac{\pi}{2} \frac{x}{L}\right) q_{u}(t)
$$

, avec $p(t)$, la position de la face émettrice en extrémité de contre-masse, fonction sinusoïdale du temps à la pulsation de résonance :

$$
\omega_{r}=\frac{\pi}{2 L} \sqrt{\frac{c}{\rho}}
$$

En supposant que la déformée conserve cette expression autour de la résonance du transducteur; le Lagrangien du Langevin est fonction uniquement de la position de la face émettrice $q_{u}(t)$, de la vitesse vibratoire $\dot{q}_{u}(t)$ et de la tension d'alimentation $v(t)$ :

$$
L\left(\dot{q}_{u}, q_{u}, v\right)=\frac{1}{2} \int\left(\rho \dot{u}^{2}-T S+E D\right) d \Omega+\sum f p-v q_{c}
$$

, avec :

- $f$, la force qu'exerce la charge sur la face émettrice ;

- $q_{c}$, la charge électrique fournit par l'alimentation.

Le Lagrangien du transducteur s'exprime en sommant les différentes énergies mises en jeu. L'énergie cinétique s'exprime par :

$$
T=\frac{1}{2} 2 \int_{L_{c e r}}^{L} A \rho \dot{u}(x)^{2} d x=\frac{1}{2} M \dot{q}_{u}^{2}
$$

avec :

$$
M=2 \int_{0}^{L} A \rho \sin \left(\frac{\pi}{2} \frac{x}{L}\right)^{2} d x=A \rho L
$$

L'énergie élastique des contre-masses s'exprime par :

$$
V_{C M}=\frac{1}{2} 2 \int_{L_{c e r}}^{L} A c\left(\frac{\partial u(x)}{\partial x}\right)^{2} d x=\frac{1}{2} K_{C M} q_{u}{ }^{2}
$$

avec :

$$
K_{C M}=2 \int_{L_{c e r}}^{L} A c\left(\frac{\pi}{2 L}\right)^{2} \cos \left(\frac{\pi}{2} \frac{x}{L}\right)^{2} d x=A c\left(\frac{\pi}{2 L}\right)^{2}\left(L-L_{c e r}-\frac{L}{\pi} \sin \left(\pi \frac{L_{c e r}}{L}\right)\right)
$$

Les céramiques comportent quant à elles un terme d'énergie électrique couplée à l'énergie élastique de déformation. Les équations locales liant $T, S, D$ et $E$ sont exprimées en fonction de $D$ (constant sur l'épaisseur de la céramique) et de $S$ (connue par l'expression de $u(x)$ ):

$$
\left\{\begin{array}{l}
T=c^{D} S-h D \\
E=-h S+\beta^{s} D
\end{array}\right.
$$


$D$ est constant sur l'épaisseur de la céramique (par de charge libre $\operatorname{div}(D)=0$ ). D est donc égal à sa valeur moyenne :

$$
D=<D>=\frac{<E>+h<S>}{\beta^{S}}=\frac{V}{\beta^{S} L_{c e r}}+h \frac{u\left(L_{c e r}\right)-u(0)}{\beta^{S} L_{c e r}}
$$

D'où :

$$
\begin{aligned}
& \frac{1}{2} \int(E D-T S) d \Omega \\
& =\frac{1}{2} \int\left(\beta^{S} D^{2}-c^{D} S^{2}\right) d \Omega \\
& =\frac{1}{2} 2 \int_{0}^{L_{c e r}} \frac{A}{\beta^{S}}\left(\frac{V}{L_{c e r}}+h \frac{u\left(L_{c e r}\right)-u(0)}{L_{c e r}}\right)^{2}-A c^{D}\left(\frac{\partial u(x)}{\partial x}\right)^{2} d x \\
& =\frac{1}{2} C_{0} V^{2}-\frac{1}{2} K_{c e r} q_{u}{ }^{2}+N q_{u} V
\end{aligned}
$$

avec :

$$
\begin{gathered}
C_{0}=2 \int_{0}^{L_{c e r}} \frac{\beta^{S}}{L_{c e r}{ }^{2}} d x=2 \frac{A}{\beta^{S} L_{c e r}} \\
K_{c e r}=2 \int_{0}^{L_{c e r}} A c^{D}\left(\frac{\pi}{2 L}\right)^{2} \cos \left(\frac{\pi}{2} \frac{x}{L}\right)^{2}-\frac{A h^{2}}{\beta^{S} L_{c e r}{ }^{2}} \sin \left(\frac{\pi}{2} \frac{L_{c e r}}{L}\right)^{2} d x \\
=A c\left(\frac{\pi}{2 L}\right)^{2}\left(L_{c e r}+\frac{L}{\pi} \sin \left(\pi \frac{L_{c e r}}{L}\right)\right)-2 \frac{A h^{2}}{\beta^{S} L_{c e r}} \sin \left(\frac{\pi}{2} \frac{L_{c e r}}{L}\right)^{2} \\
N=2 \int_{0}^{L_{c e r}} \frac{A h \beta^{S}}{L_{c e r}^{2}} \sin \left(\frac{\pi}{2} \frac{L_{c e r}}{L}\right) d x=2 \frac{A h}{\beta^{S} L_{c e r}} \sin \left(\frac{\pi}{2} \frac{L_{c e r}}{L}\right)
\end{gathered}
$$

Le Lagrangien de l'ensemble s'exprime donc par :

$$
\begin{aligned}
& L=\frac{1}{2} \int\left(\rho \dot{u}^{2}-T S+E D\right) d \Omega+\sum f_{j}^{e} q_{j}-v q_{c} \\
& =\frac{1}{2} M \dot{q}_{u}{ }^{2}-\frac{1}{2} K q_{u}{ }^{2}+N q_{u} V+\frac{1}{2} C_{0} V^{2}+F q_{u}-V q_{c}
\end{aligned}
$$

avec : $K=K_{C M}+K_{c e r}$.

L'équation mécanique du transducteur s'obtient en dérivant le Lagrangien selon $q_{u}$. Les efforts aux extrémités des contres-masses sont notés $F$.

$$
\frac{\partial}{\partial t} \frac{L}{\partial \dot{q}_{u}}-\frac{\partial L}{\partial q_{u}}=F
$$


RIGE - proposition d'article

$$
M \ddot{q}_{u}+K q_{u}=N V+F
$$

Les pertes mécaniques peuvent être prises en compte par un terme proportionnel à la vitesse vibratoire $\dot{q}_{u}$.

$$
M \ddot{q}_{u}+C \dot{q}_{u}+K q_{u}=N V+F
$$

L'équation électrique s'obtient en dérivant selon $V$. L'effort correspondant ici à $F$ est la charge $q_{c}$ fournie par l'alimentation.

$$
\begin{gathered}
\frac{\partial}{\partial t} \frac{L}{\partial \dot{V}}-\frac{\partial L}{\partial V}=-q_{c} \\
q_{c}=N q_{u}+C_{0} V
\end{gathered}
$$

$\mathrm{Si}$ on suppose pour la suite que le transducteur est réalisé avec des céramiques d'épaisseurs faibles par rapport à la longueur des contre-masses $\left(L_{c e r}<<L\right)$, on obtient :

$$
\begin{gathered}
K=\left(\frac{\pi}{2}\right)^{2} \frac{A c}{L} \\
M=A \rho L \\
C_{0}=\frac{2 A}{\beta^{S} L_{c e r}} \\
N=\pi \frac{A h}{\beta^{S} L}=\pi \frac{A}{L} e
\end{gathered}
$$

Calcul numérique à l'aide d'ANSYS

Les calculs numériques, à l'aide de logiciels par éléments finis, généralement rencontrés dans la littérature, portent sur le calcul des modes et des fréquences de résonances, de l'admittance du stator seul ou de la détermination du schéma équivalent à l'aide du calcul de la résonance et de l'antirésonance [BAO 00]. Ces derniers ne caractérisent cependant pas de manière complète la conversion électromécanique de l'actionneur et peuvent être relativement long en temps de calcul (balayage en fréquence d'une excitation harmonique). La méthode proposée ici ne nécessite qu'un calcul de mode de résonance et permet, à l'aide de l'évaluation des énergies cinétiques et élastiques, de déterminer les paramètres introduits précédemment. Le logiciel utilisé pour illustrer cette méthode est ANSYS r5.7. Les différentes étapes à mener sont les suivantes :

- Dans le module Preprocessor : définir la géométrie, les matériaux et le maillage du système ;

- Dans le module Solution: demander une nouvelle analyse de type Modale (par la méthode de Block-Lanczos) en précisant de calculer les résultats aux éléments (activation du calcul des données énergétiques) et en court-circuitant les céramiques ; 
- Dans le module Postprocessor: sélectionner le mode désiré et évaluer les énergies cinétiques $T$ et élastiques $V$, la déformée $q_{u}$ pour laquelle ces dernières sont calculer et les charges résultantes $q_{c}$ sur une électrode.

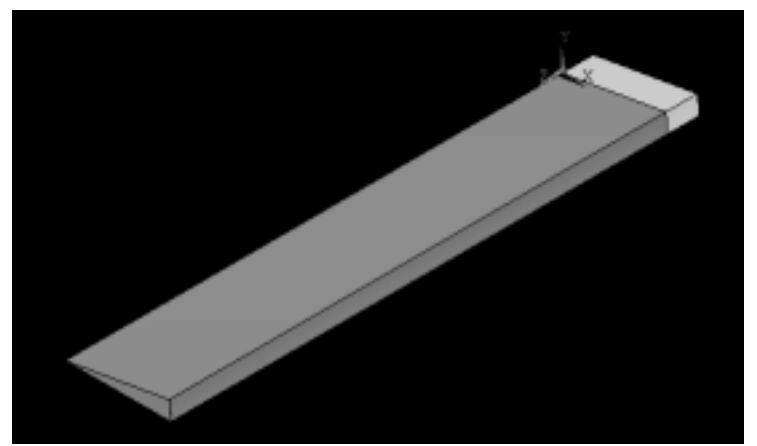

Figure 3. Géométrie du transducteur Langevin pour la simulation (1/72 ième)

Pour le mode de résonance considéré (à la pulsation $\omega$ ), le tableau suivant résume les étapes à réaliser pour obtenir les paramètres recherchés :

\begin{tabular}{|c|c|c|}
\hline Grandeur obtenue par Ansys & Commandes Ansys & Paramètre associé \\
\hline $\begin{array}{l}\text { Amplitude de la déformée } \\
\qquad q_{u}\end{array}$ & $\begin{array}{c}\text { Plot Results / Nodal Solution / } \\
\text { Translation }\end{array}$ & \\
\hline $\begin{array}{c}\text { Energie cinétique } \\
T=\frac{1}{2} M \dot{\mathrm{q}}_{\mathrm{u}}{ }^{2}=\frac{1}{2} M \omega^{2} q_{u}{ }^{2}\end{array}$ & $\begin{array}{c}\text { Element Table / Sum of each } \\
\text { item } \\
\text { aprè avoir défini dans la table } \\
\text { Element l'énergie cinétique } \\
\text { Kene }\end{array}$ & $\begin{array}{l}\text { Masse modale } M \\
\qquad M=\frac{2 T}{\omega^{2} p^{2}}\end{array}$ \\
\hline $\begin{array}{l}\text { Energie élastique } \\
\qquad V=\frac{1}{2} k q_{u}{ }^{2}\end{array}$ & $\begin{array}{l}\text { Idem que pour } T \text { avec ici } \\
\text { l'énergie de déformation Sene }\end{array}$ & $\begin{array}{l}\text { Raideur modale } \\
\qquad K=\frac{2 V}{q_{u}^{2}}\end{array}$ \\
\hline $\begin{array}{l}\text { Charge sur une électrode } \\
\qquad q_{c}\end{array}$ & $\begin{array}{l}\text { Nodal Calcs / Total Sum } \\
\text { Force } \\
\text { sur les noeuds de l'électrode }\end{array}$ & $\begin{array}{l}\text { Facteur d'effort } \\
N=\frac{q_{c}}{q_{u}}(V \text { est } \\
\text { nul })\end{array}$ \\
\hline
\end{tabular}

Pour un transducteur Langevin de longueur totale $10 \mathrm{~cm}(2 L)$, de diamètre $2 \mathrm{~cm}$, d'épaisseur de céramique (P191 de Quartz et Silice) $6 \mathrm{~mm}\left(2 L_{c e r}\right)$, les résultats obtenus sont présentés dans le tableau suivant :

\begin{tabular}{|c|c|c|}
\hline & Analytique & ANSYS \\
\hline Fréquence de résonance & $18,898 \mathrm{kHz}$ & $18,617 \mathrm{kHz}$ \\
\hline Raideur K & $1,7010^{9}$ & $1,6210^{9}$ \\
\hline
\end{tabular}


RIGE - proposition d'article

\begin{tabular}{|c|c|c|}
\hline Masse vibrante M & $121 \mathrm{~g}$ & $118,1 \mathrm{~g}$ \\
\hline Facteur d'effort N & $0,28 \mathrm{~N}_{\mathbf{N}} \mathrm{V}^{-1}$ & $0,283 \mathrm{~N}^{-\mathrm{V}^{-1}}$ \\
\hline
\end{tabular}

Il y a donc une bonne corrélation entre les résultats obtenus de manière analytique ou numérique. Un maillage fin est cependant nécessaire au niveau du logiciel par éléments finis. Il est également possible de comparer les déformées et les contraintes obtenues. La modélisation numérique permet également d'obtenir la répartition des contraintes (notamment au niveau des céramiques).

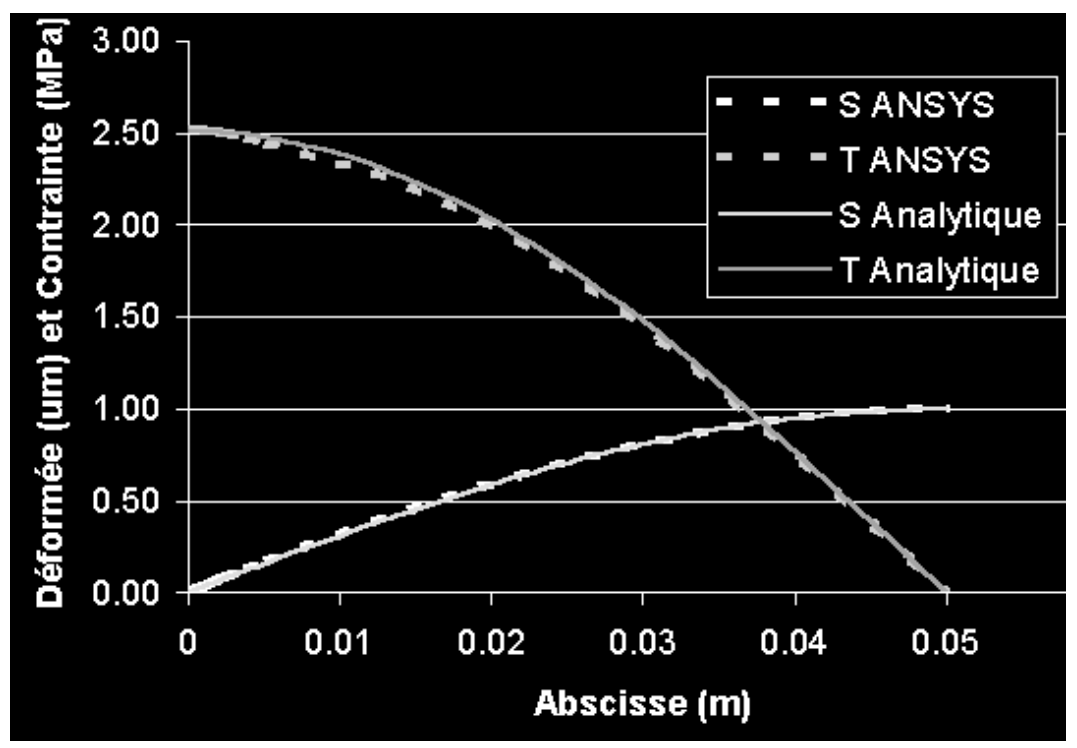

Figure 4. Répartitions de déformation et de contrainte

\section{Calcul des paramètres électromécaniques du stator du moteur}

\subsection{Principe et structure du moteur}

Un moteur à rotation de mode utilise les 2 modes de flexion orthogonaux d'une poutre cylindrique composée de céramiques piézo-électriques encadrées par des contre masses [4][5]. Le tout est précontraint par une vis. La partie mobile est plaquée contre l'extrémité du cylindre par un ressort. Les céramiques utilisées pour exciter le stator du moteur sont des disques avec des polarisations opposées sur chaque moitié. Elles sont disposées orthogonalement pour exciter chacune un mode de flexion. Elles sont alimentées par des sources de tensions sinusoïdales hautes fréquences (plusieurs dizaines de $\mathrm{kHz}$ ) en quadrature de manière à obtenir une onde progressive en extrémité de contre-masse. Outre sa simplicité structurelle et les conditions favorables qu'il permet d'assurer au niveau du contact rotor/stator (contact permanent à pression répartie), ce moteur présente aussi l'avantage d'une 
structure empilée précontrainte qui évite les opérations coûteuses et délicates de collage.

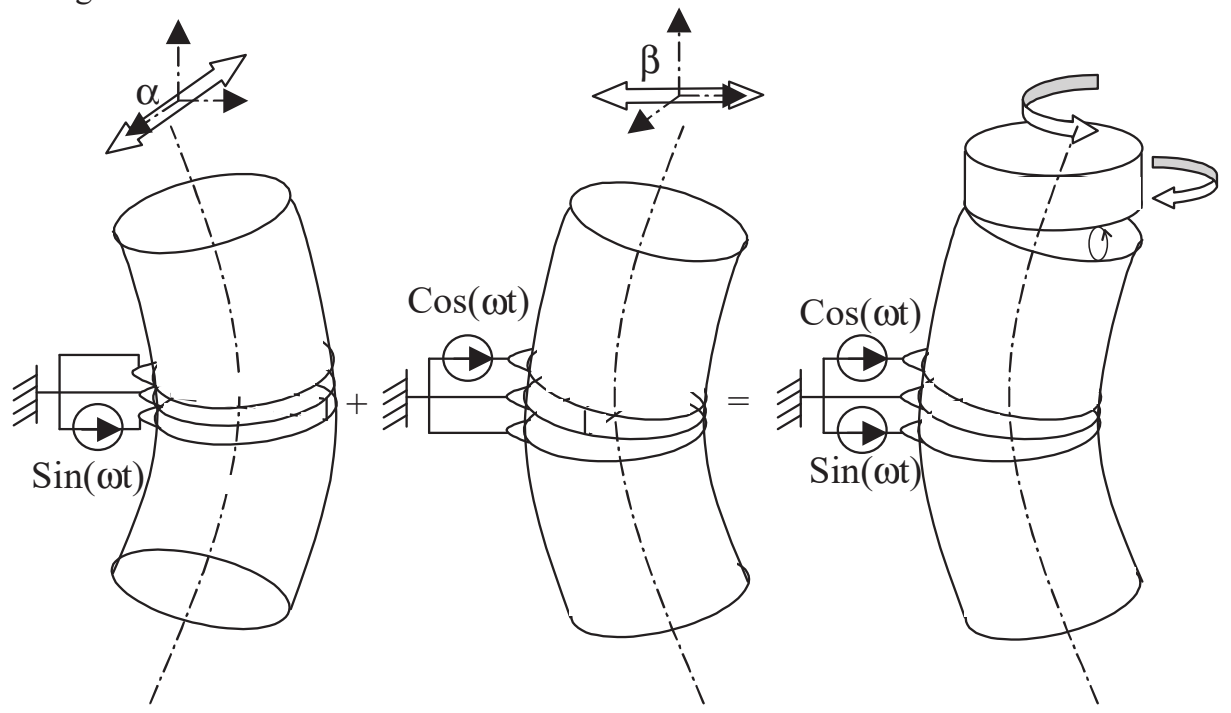

Figure 5. Obtention d'une onde progressive

\subsection{Modélisation analytique du stator}

Deux exemples de géométries de stator seront traités : un stator de moteur de petite taille avec des contre-masse en acier (pour comparer résultats numériques et mesures), un stator de plus grande taille avec des contre-masse aux propriétés mécaniques similaires à celle des céramiques (pour comparer résultats analytiques et numériques).

\section{Approximation de la déformée}

Pour la modélisation simplifiée, les céramiques et les contre-masses sont supposées avoir les mêmes caractéristiques mécanique (élasticité et densité). De la même manière que pour le transducteur Langevin, il est possible d'établir l'équation de mouvement de la poutre en flexion constituant le stator. Les déformées, efforts et grandeurs géométriques caractéristiques de la déformées sont représentés figure 6 : section $A$, moment d'inertie $I$, déplacements orthogonaux $u$, rotation de section $\Psi$, effort de cisaillement $T$, moment $M$. Le principe fondamentale de la dynamique et les équations élastique sur cette tranche élémentaire de matériaux permettent d'obtenir le jeu d'équations suivant :

$$
\begin{aligned}
\rho A \frac{\partial^{2} u}{\partial t^{2}}=-\frac{\partial T}{\partial x} & \text { et } & \rho I \frac{\partial^{2} \Psi}{\partial t^{2}}=\frac{\partial M}{\partial x}-T \\
\frac{\partial \psi}{\partial x}=\frac{M}{c I} & \text { et } & \psi-\frac{T}{a_{T} A G}=\frac{\partial u}{\partial x}
\end{aligned}
$$




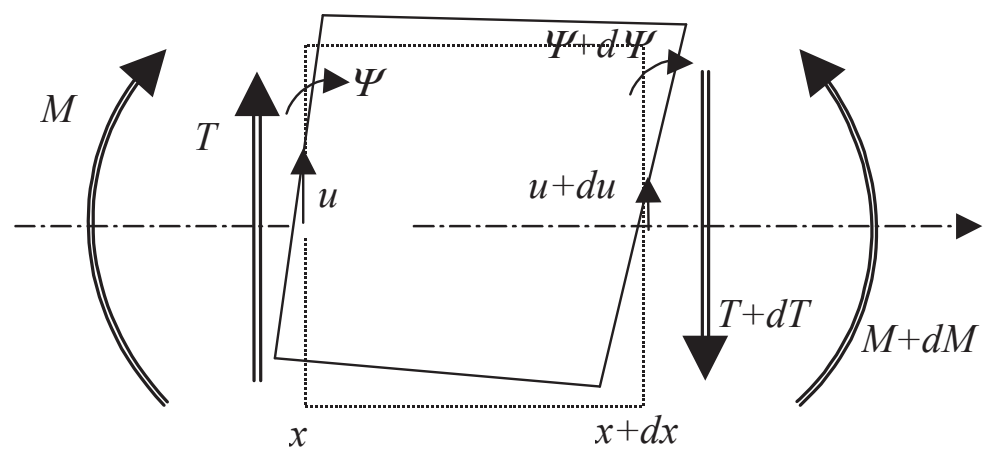

Figure 6. Elément de barre en flexion

Les poutre étudiée sont relativement courtes, l'effet de l'inertie de la section, $\rho I d x\left(\partial^{2} \Psi / \partial t^{2}\right)$, et du cisaillement, $T /\left(a_{T} S G\right)$, ne peuvent être négligés [TIM]. L'équation d'onde prend alors la forme suivante :

$$
c I \frac{\partial^{4} u}{\partial x^{4}}+\frac{\rho^{2} I}{a G} \frac{\partial^{4} u}{\partial t^{4}}+\rho S \frac{\partial^{2} u}{\partial t^{2}}=\left(\rho I+\frac{c \rho I}{a G}\right) \frac{\partial^{4} u}{\partial t^{2} \partial x^{2}}
$$

En notation complexe, cette équation différentiel admet pour solution $U(x)$ dont on peut déduire les expressions de $M(x), \Psi(x)$ et $T(x)$. Le stator étudié ici a une géométrie symétrique selon l'axe longitudinal. Les déformées sont de plus normées par rapport au déplacement $q_{u}(t)$ de la ligne neutre en extrémité du stator. Les expressions des déformées peuvent donc s'exprimer par :

$$
\begin{aligned}
u(x, t) & =U(x) q_{u}(t) \\
\psi(x, t) & =\Psi(x) q_{u}(t)
\end{aligned}
$$

avec $U(L / 2)=1$. Le tableau suivant résume ces différentes expressions :

\begin{tabular}{|l|r|}
\hline$U(x)=A \cos \left(k_{1} x\right)+B \operatorname{ch}\left(k_{2} x\right)$ & $k_{1}=\sqrt{\frac{\sqrt{\Delta}+\rho I\left(1+\frac{c}{a_{T} G}\right) \omega^{2}}{2 c I}}$ \\
$k_{2}=\sqrt{\frac{\sqrt{\Delta}-\rho I\left(1+\frac{c}{a_{T} G}\right) \omega^{2}}{2 c I}}$ \\
$\Delta=\left(\rho I\left(1+\frac{c}{a_{T} G}\right) \omega^{2}\right)^{2}+$ \\
$4 c I \rho S \omega^{2}\left(1-\frac{\rho I}{a_{T} S G} \omega^{2}\right)$
\end{tabular}




\begin{tabular}{|l|l|}
\hline$M(x)=\alpha_{M} A \cos \left(k_{1} x\right)+\beta_{M} B c h\left(k_{2} x\right)$ & $\alpha_{M}=c I\left(\frac{\rho \omega^{2}}{a_{T} G}-k_{1}^{2}\right)$ \\
& $\beta_{M}=c I\left(\frac{\rho \omega^{2}}{a_{T} G}+k_{2}^{2}\right)$ \\
\hline$\Psi(x)=-\alpha_{\psi} A \sin \left(k_{1} x\right)+\beta_{\psi} B \operatorname{sh}\left(k_{2} x\right)$ & $\alpha_{\psi}=\frac{k_{1}\left(\alpha_{M}+a_{T} S G\right)}{a_{T} S G-\rho I \omega^{2}}$ \\
& $\beta_{\psi}=\frac{k_{2}\left(\beta_{M}+a_{T} S G\right)}{a_{T} S G-\rho I \omega^{2}}$ \\
\hline$T(x)=-\alpha_{T} A \sin \left(k_{1} x\right)+\beta_{T} B \operatorname{sh}\left(k_{2} x\right)$ & $\beta_{T}=a_{T} S G\left(\beta_{\psi}-k_{2}\right)$ \\
$\alpha_{T}=a_{T} S G\left(\alpha_{\psi}-k_{1}\right)$ \\
\hline
\end{tabular}

Les coefficient $k_{1}$ et $k_{2}$ sont connus, reste à déterminer $A$ et $B$ qui doivent respecter les conditions suivantes :

$$
U(L / 2)=1 \text { et } T(L / 2)=0
$$

D'où les expressions :

$$
B=\frac{1}{\frac{\beta_{T}}{\alpha_{T}} \frac{\operatorname{sh}\left(k_{2} L / 2\right)}{\tan \left(k_{1} L / 2\right)}+\operatorname{ch}\left(k_{2} L / 2\right)} \text { et } A=B \frac{\beta_{T}}{\alpha_{T}} \frac{\operatorname{sh}\left(k_{2} L / 2\right)}{\sin \left(k_{1} L / 2\right)}
$$

On obtient donc l'expression des déformées :

en posant :

$$
\begin{aligned}
& U(x)=A \cos \left(k_{1} x\right)+B \operatorname{ch}\left(k_{2} x\right) \\
& \Psi(x)=C \sin \left(k_{1} x\right)+D \operatorname{sh}\left(k_{2} x\right)
\end{aligned}
$$

$$
C=-\alpha_{\psi} A \text { et } D=\beta_{\psi} B
$$

\section{Expression du Lagrangien}

Comme pour l'exemple du transducteur Langevin, le calcul du Lagrangien du stator passe par le calcul de chacune de ses composantes énergétiques. L'énergie cinétique $T$ du stator s'exprime ainsi par :

$$
\begin{gathered}
T=\frac{1}{2} \int \rho v^{2} d \Omega=\frac{1}{2} \int \rho I \dot{\psi}(x, t)^{2}+\rho \operatorname{Si}(x, t)^{2} d x=\frac{1}{2} M \dot{q}_{u}(t)^{2} \\
M=\int \rho I \Psi(x)^{2}+\rho S U(x)^{2} d x \text { avec } I=\iint y^{2} d A
\end{gathered}
$$

Pour les contre-masses, l'énergie potentielle $V$ n'est constitué que d'un potentiel de déformation élastique. Dans le cas du moteur à rotation de mode, les contraintes en cisaillement ne sont pas négligées, et l'expression de $V$ est de la forme : 
RIGE - proposition d'article

$$
\begin{aligned}
& V=\frac{1}{2} \int T S d \Omega \\
& =\frac{1}{2} \int E I\left(\frac{\partial \psi(x, t)}{\partial x}\right)^{2}+a S G\left(-\psi(x, t)+\frac{\partial u(x, t)}{\partial x}\right)^{2} d x \\
& =\frac{1}{2} K_{C M} q_{u}(t)^{2} \\
& K_{C M}=\int E I\left(\frac{\partial \Psi(x)}{\partial x}\right)^{2}+a S G\left(-\Psi(x)+\frac{\partial U(x)}{\partial x}\right)^{2} d x
\end{aligned}
$$

Pour les céramiques, le potentiel $V$ comporte en plus un terme énergétique d'origine électrique :

$$
V_{c e r}=\frac{1}{2} \int(T S-E D) d \Omega
$$

Le jeu d'équations locales utilisé est le même que pour le transducteur Langevin. $D$ est constant sur l'épaisseur de la céramiques, mais est variable selon la position $y$ (figure 7) à cause de la déformation $S=\frac{\partial \Psi(x)}{\partial x} y$ non constante :

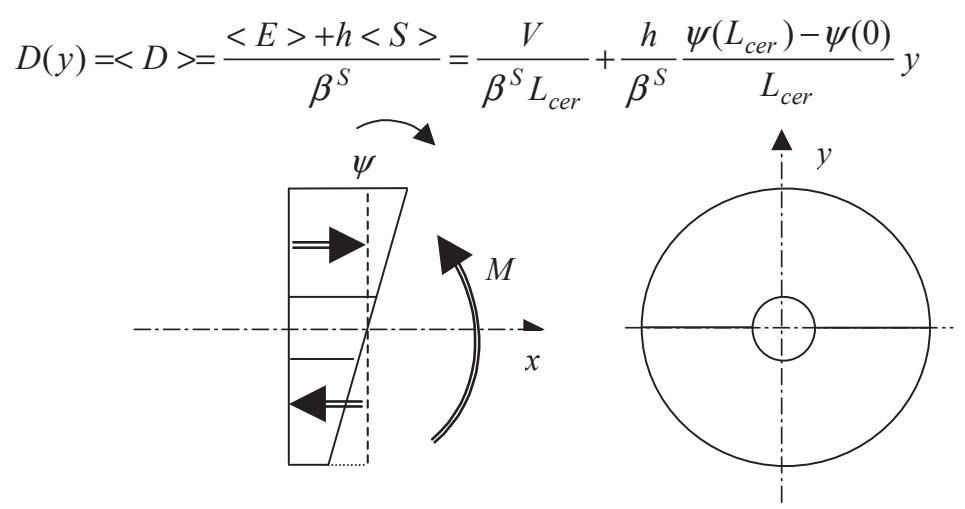

Figure 7. Déformation d'une céramique du stator sous l'action d'une tension

D'où l'expression de $V$ :

$$
\begin{gathered}
V_{c e r}=\frac{1}{2} \int\left(-\beta^{S} D^{2}+c^{D} S^{2}\right) d \Omega \\
=\frac{1}{2} 2 \int_{0}^{L_{c e r}}-\beta^{S} A D^{2}+c^{D} I\left(\frac{\partial \Psi(x)}{\partial x}\right)^{2}+a S G\left(-\Psi(x)+\frac{\partial U(x)}{\partial x}\right)^{2} d x \\
V_{c e r}=\frac{1}{2} K_{c e r} q_{u}{ }^{2}-\frac{1}{2} C_{0} v^{2}-N q_{u} v
\end{gathered}
$$

avec 


$$
\begin{gathered}
C_{0}=\int \frac{\varepsilon^{s} A}{L^{2}} d x=2 \frac{\varepsilon^{s} A}{L_{c e r}} \\
k_{C E R}=\int-h^{2} \varepsilon^{S} I \frac{\psi\left(L_{c e r}\right)^{2}}{L_{c e r}}+c^{D} I\left(\frac{\partial \Psi(x)}{\partial x}\right)^{2}+a S G\left(-\psi(x)+\frac{\partial U(x)}{\partial x}\right)^{2} d x \\
N=\frac{1}{2} \int 2 h \mathcal{E}^{s} I_{p} \frac{\Psi\left(L_{c e r}\right)}{L_{c e r}{ }^{2}} d x \text { avec } I_{p}=\iint|y| d A
\end{gathered}
$$

Etablissement des équations mécaniques et électriques

Le stator est utilisé selon deux modes résonances, $\alpha$ et $\beta$, orthogonaux et de fréquence identique. Pour des actions et des déplacements selon un mode $\alpha$, le Lagrangien obtenu est de la forme :

$$
L_{\alpha}=\frac{1}{2} M \dot{q}_{u \alpha}{ }^{2}-\frac{1}{2} K q_{u \alpha}{ }^{2}+N q_{u \alpha} v_{\alpha}+\frac{1}{2} C_{0} v_{\alpha}{ }^{2}+f_{\alpha} q_{u \alpha}-v_{\alpha} q_{c \alpha}
$$

avec : $q_{u \alpha}(t)=q_{u}(t)$ et $v_{\alpha}(t)=v(t)$. Le Lagrangien selon le mode $\beta$ est de la même forme. En dérivant le Lagrangien les paramètres $w$, on obtient l'équation mécanique :

$$
\left(\begin{array}{cc}
M & 0 \\
0 & M
\end{array}\right)\left(\begin{array}{l}
\ddot{q}_{u \alpha} \\
\ddot{q}_{u \beta}
\end{array}\right)+\left(\begin{array}{cc}
K & 0 \\
0 & K
\end{array}\right)\left(\begin{array}{l}
q_{u \alpha} \\
q_{u \beta}
\end{array}\right)=\left(\begin{array}{cc}
N & 0 \\
0 & N
\end{array}\right)\left(\begin{array}{l}
v_{\alpha} \\
v_{\beta}
\end{array}\right)+\left(\begin{array}{c}
f_{\alpha} \\
f_{\beta}
\end{array}\right)
$$

avec : $K=K_{C M}+K_{C E R}$. On peut ajouter a cette équation un terme de perte mécanique proportionnel à la vitesse vibratoire.

En dérivant le Lagrangien selon les paramètres $v$, on obtient l'équation électrique :

$$
\left(\begin{array}{l}
q_{c \alpha} \\
q_{c \beta}
\end{array}\right)=\left(\begin{array}{cc}
N & 0 \\
0 & N
\end{array}\right)\left(\begin{array}{l}
q_{u \alpha} \\
q_{u \beta}
\end{array}\right)+\left(\begin{array}{cc}
C_{0} & 0 \\
0 & C_{0}
\end{array}\right)\left(\begin{array}{l}
v_{\alpha} \\
v_{\beta}
\end{array}\right)
$$

\subsection{Comparaison des résultats analytiques et numériques}

Le principe de calcul est identique à celui présenté pour le transducteur Langevin. La géométrie simulée ici est représentée figure 8 . 


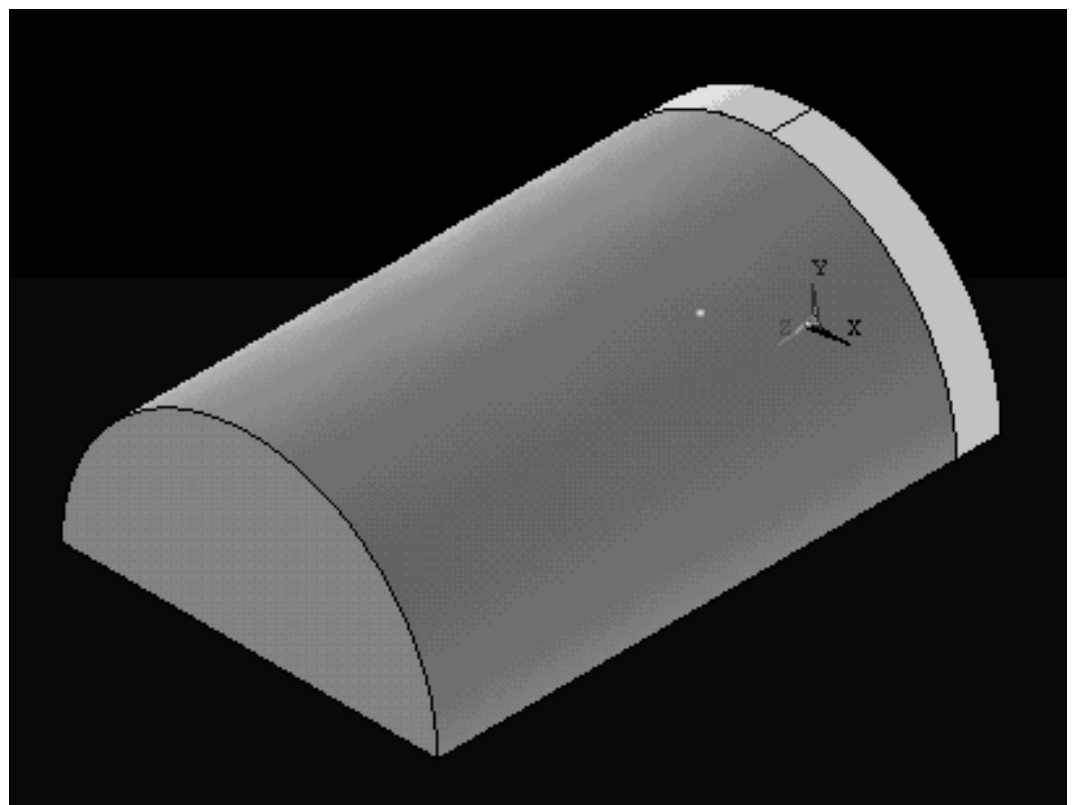

Figure 8. Géométrie du stator pour la simulation (1/8 ième)

Pour un actionneur à rotation de mode de longueur totale $150 \mathrm{~mm}(2 L)$, de diamètre extérieur $5 \mathrm{~cm}$, d'épaisseur de céramique (P189 Quartz et Silice) $6 \mathrm{~mm}\left(2 L_{c e r}\right)$, les résultats obtenus sont présentés dans le tableau suivant :

\begin{tabular}{|c|c|c|}
\hline & Analytique & ANSYS \\
\hline Fréquence de résonance & $5.2 \mathrm{kHz}$ & $5.268 \mathrm{kHz}$ \\
\hline Raideur K & $7.910^{8}$ & $7.710^{8}$ \\
\hline Masse vibrante $\mathbf{M}$ & $750 \mathrm{~g}$ & $730 \mathrm{~g}$ \\
\hline Facteur d'effort $\mathbf{N}$ & 0.38 & 0.40 \\
\hline
\end{tabular}

On trouve une très bonne corrélation entre les résultats analytiques et numériques (moins de $5 \%$ d'écart) tant au niveau des paramètres électromécaniques que des déformées (figure 9). Le modèle analytique considéré est d'autant plus valable que la poutre est longue et la raideur des matériaux utilisés importantes. Pour un stator de longueur $100 \mathrm{~mm}$, les résultats différent de 15 à $20 \%$. Les déformées sont alors moins bien approximées par le modèle de poutre. Cette dernière géométrie ,avec un rapport longueur sur diamètre de 2 et un matériau au module d'Young assez faible $(80 \mathrm{GPa})$, constitue un cas limite du modèle analytique. 
Modélisation d'un moteur piézoélectrique 17

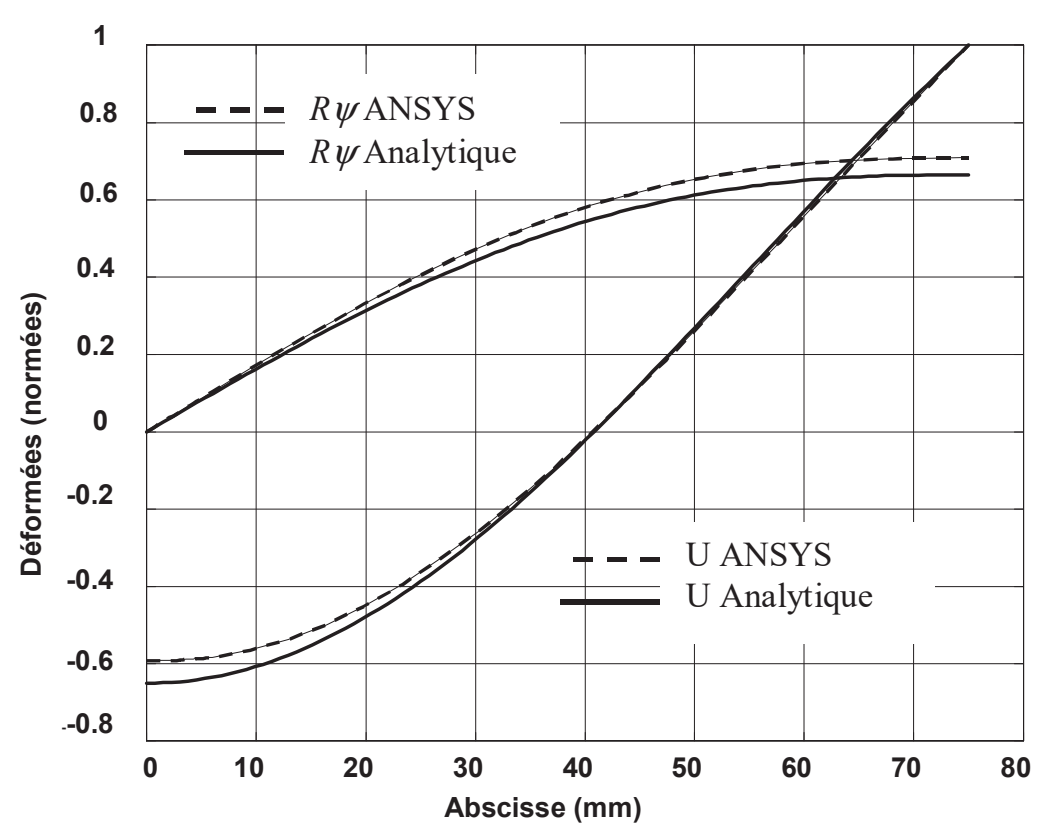

Figure 9. Répartitions des déformations

\subsection{Comparaison des résultats numériques et des mesures}

L'exemple suivant est celui du stator d'un prototype de moteur à rotation de mode diphasé constitué de :

- 2 contre-masse en acier de $20 \mathrm{~mm}$ de long et $25 \mathrm{~mm}$ de diamètre;

- 2 phases orthogonales de 4 céramiques PC4 (Morgan Matroc) (0,5 mm d'épaisseur pour chaque céramique) ; 


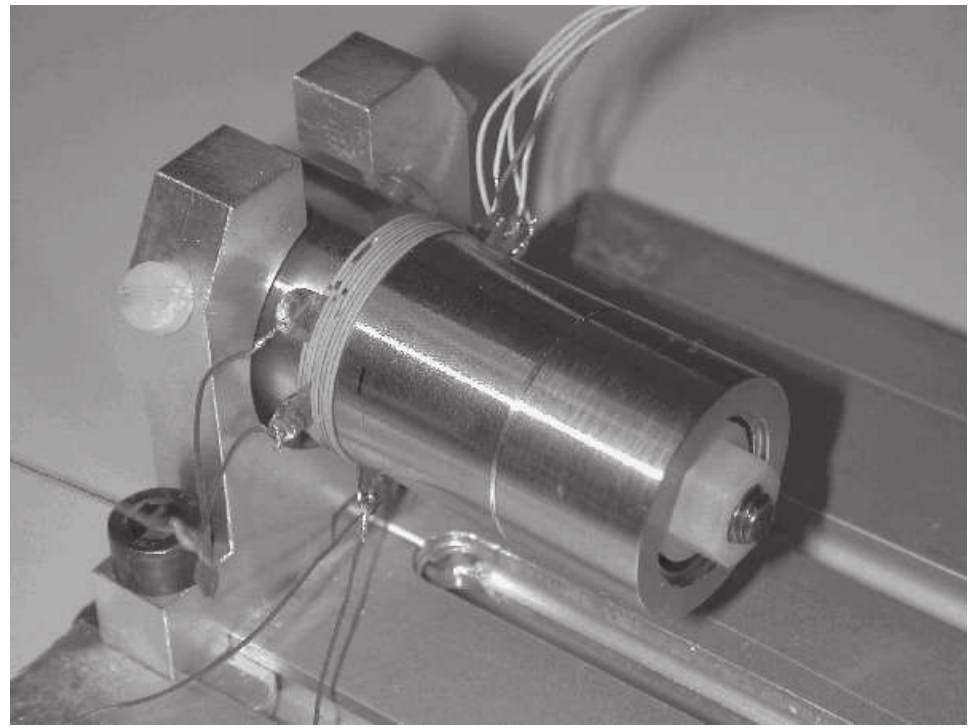

Figure 10. Moteur à rotation de mode

Une mesure d'admittance effectuées sur une phase alimentée sous $5 V_{\max }$, l'autre étant court-circuitée, permet d'obtenir le circuit équivalent d'une phase du stator à vide. Ce circuit est représenté figure 11.

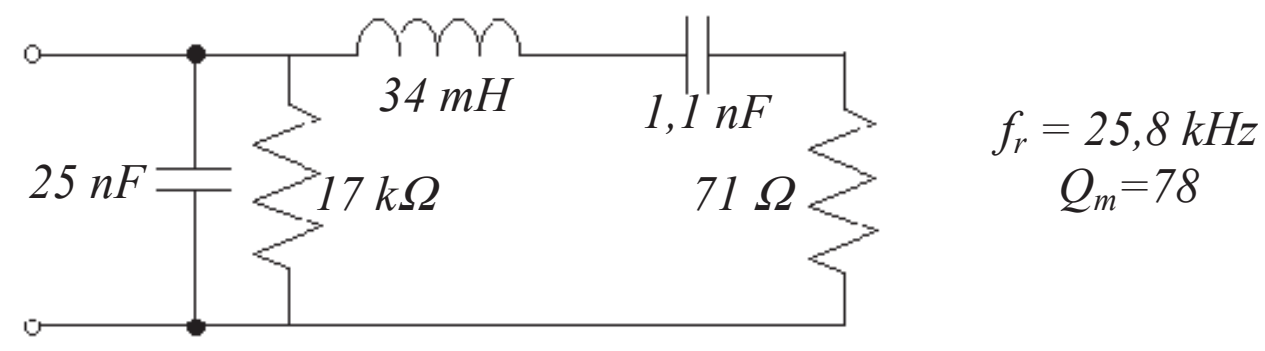

Figure 11. Circuit équivalent obtenu à partir de la mesure d'admittance

Pour obtenir le facteur d'effort $N$ du moteur, une mesure de la vitesse vibratoire sur la déformée orthogonale en extrémité de contre-masse. Ce facteur d'effort permet de calculer par les suite les masses et raideurs du mode mécanique considéré. Les résultats obtenus sont résumés dans le tableau suivant :

\begin{tabular}{|c|c|c|c|}
\hline & Mesures & ANSYS & Ecart \\
\hline $\begin{array}{c}\text { Fréquence de } \\
\text { résonance }\end{array}$ & $25,8 \mathrm{kHz}$ & $32,4 \mathrm{kHz}$ & $20 \%$ \\
\hline Rapport $\boldsymbol{U} / \boldsymbol{R} \boldsymbol{\psi}$ & 1,05 & 1,045 & $0,5 \%$ \\
\hline
\end{tabular}




\begin{tabular}{|c|c|c|c|}
\hline Masse $\boldsymbol{M}$ & $55 \mathrm{~g}$ & $60 \mathrm{~g}$ & $8 \%$ \\
\hline Raideur $\boldsymbol{K}$ & $1,45.10^{9} \mathrm{~N} / \mathrm{m}$ & $2,5010^{9} \mathrm{~N} / \mathrm{m}$ & $40 \%$ \\
\hline Dissipation $\boldsymbol{C}$ & $114 \mathrm{~N} / \mathrm{m}^{-1}$ & $122^{(1)} \mathrm{N} / \mathrm{m}^{-1}$ & $7 \%$ \\
\hline Facteur d'effort $\boldsymbol{N}$ & $1,27 \mathrm{~N} / \mathrm{V}$ & $1,52 \mathrm{~N} / \mathrm{V}^{(1)}$ & $16 \%$ \\
& & $1,31 \mathrm{~N} / \mathrm{V}^{(2)}$ & $3 \%$ \\
\hline
\end{tabular}

(1) Valeur calculée en prenant un coefficient de qualité mécanique de 100.

${ }^{(2)}$ Valeur calculée en prenant en compte une surface inactive de céramique.

On trouve une relativement bonne corrélation entre les résultats de simulation et de mesures. L'écart principal se fait sur la raideur globale de la structure. La simulation surestime cette dernière de $40 \%$. L'écart est peut-être due à une prise en compte incomplètes des interfaces entre les différents éléments (elles sont supposées parfaites en simulation).

\section{Prise en compte du contact et obtention des caractéristiques mécaniques du moteur}

Les étapes présentées dans les paragraphes précédents ont permis d'établir un modèle matriciel simple de la conversion électromécanique et du comportement dynamique du stator. Pour obtenir un modèle complet et global du moteur, il faut prendre en compte la conversion d'énergie mécano-mécanique par frottements qui permet de transformer les vibrations microscopiques du stator en un mouvement continu de rotation du rotor. Cette étape est réalisée de manière analytique, une approche numérique étant très lourde à mettre en œuvre.

\subsection{Cinématique du contact}

Avant de calculer les efforts modaux $f$ correspondant à l'action du rotor sur le stator lors du contact induit par le passage de l'onde progressive, il faut exprimer ces mouvements vibratoires et la cinématique des points matériels du stator participant au contact. Le stator se déforme selon deux modes $\alpha$ et $\beta$. Ces déformées ont été normées selon le déplacement orthogonal $U$ en extrémité de contre-masse . La déformée angulaire $\psi$ en cette section est également un paramètre important. Elle est liée à $U$ par une relation linéaire fonction de la géométrie du stator et calculable par les expressions établies précédemment. 

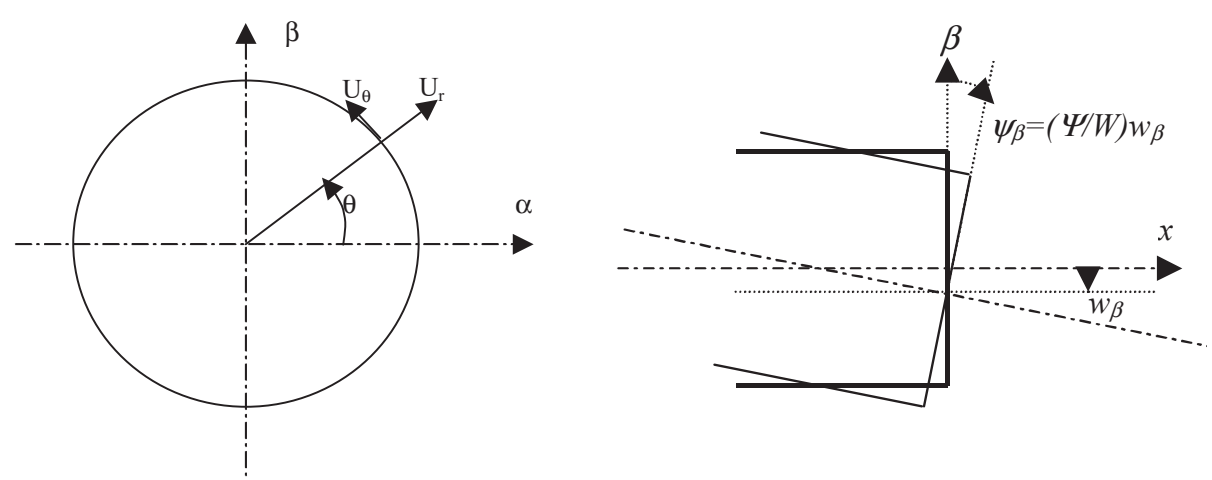

Figure 3. Déformation du stator au niveau du contact

Dans le repère $\left(\mathrm{U}_{\alpha}, \mathrm{U}_{\beta}, \mathrm{U}_{\mathrm{x}}\right)$ les déformées sont :

\begin{tabular}{|c|c|c|}
\hline Phase $\alpha$ alimentée & Phase $\beta$ alimentée & Phases $\alpha$ et $\beta$ alimentées \\
\hline$\left(U^{\alpha}\right)=\left(\begin{array}{c}w_{\alpha} \\
0 \\
-R_{e} \frac{\Psi}{W} \cos (\theta) w_{\alpha}\end{array}\right)$ & $\left(U^{\beta}\right)=\left(\begin{array}{c}0 \\
w_{\beta} \\
-R_{e} \frac{\Psi}{W} \sin (\theta) w_{\beta}\end{array}\right)$ & $\begin{array}{l}\left(U^{T}\right)=\left(U^{\alpha}+U^{\beta}\right) \\
=\left(\begin{array}{c}w_{\alpha} \\
w_{\beta} \\
-R_{e} \frac{\Psi}{W}\left(w_{\alpha} \cos (\theta)+w_{\beta} \sin (\theta)\right)\end{array}\right.\end{array}$ \\
\hline
\end{tabular}

Le déplacement total s'exprime donc par la somme de ces deux déplacements et donne dans le repère de coordonnées cylindriques $\left(\mathrm{U}_{\mathrm{r}}, \mathrm{U}_{\theta}, \mathrm{U}_{\mathrm{x}}\right)$ :

$$
\left(U^{T}\right)=\left(\begin{array}{c}
w_{\alpha} \cos (\theta)+w_{\beta} \sin (\theta) \\
-w_{\alpha} \sin (\theta)+w_{\beta} \cos (\theta) \\
-R_{e} \frac{\Psi}{W}\left(w_{\alpha} \cos (\theta)+w_{\beta} \sin (\theta)\right)
\end{array}\right)
$$

La position du point de contact est donnée pour la déformée maximale soit $\frac{d U_{\gamma}}{d \theta}=0$, d'où $w_{\alpha} \sin \left(\theta_{c}\right)-w_{\beta} \cos \left(\theta_{c}\right)=0$.

Les déformées en régime permanent sont en quadrature de phase et peuvent se noter:

Ce qui donne :

$$
\left(\begin{array}{l}
w_{\alpha} \\
w_{\beta}
\end{array}\right)=W\left(\begin{array}{l}
\cos (\omega t) \\
\sin (\omega t)
\end{array}\right)
$$




$$
\left(U^{T}\right)=\left(\begin{array}{c}
w_{\alpha} \cos (\theta)+w_{\beta} \sin (\theta) \\
-w_{\alpha} \sin (\theta)+w_{\beta} \cos (\theta) \\
-R_{e} \frac{\Psi}{W}\left(w_{\alpha} \cos (\theta)+w_{\beta} \sin (\theta)\right)
\end{array}\right)=\left(\begin{array}{c}
W \cos (\omega t-\theta) \\
W \sin (\omega t-\theta) \\
-R_{e} \frac{\Psi}{W}(W \cos (\omega t-\theta))
\end{array}\right)
$$

On donc bien une onde progressive. Le contact a lieu pour $\mathrm{U}_{\gamma}$ maximum soit pour $\theta_{c}=\omega t-\pi$. La vitesse particulaire est donnée par :

$$
\left(\begin{array}{c}
v_{r} \\
v_{\theta} \\
v_{x}
\end{array}\right)=\left(\begin{array}{c}
\dot{w}_{\alpha} \cos (\theta)+\dot{w}_{\beta} \sin (\theta) \\
-\dot{w}_{\alpha} \sin (\theta)+\dot{w}_{\beta} \cos (\theta) \\
-R_{e} \frac{\Psi}{W}\left(\dot{w}_{\alpha} \cos (\theta)+\dot{w}_{\beta} \sin (\theta)\right)
\end{array}\right)
$$

\subsection{Expression des efforts dans le cas d'un contact ponctuel}

Les efforts au sein du contact sont appliqués selon certaines directions. Les forces de réactions modales correspondantes s'obtiennent en projetant ces derniers selon les coordonnées généralisées à l'aide d'expressions du type :

$$
F_{r \alpha}=\sum_{i} F_{i} \frac{\partial x_{i}}{\partial w_{\alpha}}
$$

Ce qui donne :

$$
f_{\alpha}=-F_{N} \frac{\partial U \gamma}{\partial w_{\alpha}}+F_{T} \frac{\partial U_{\theta}}{\partial w_{\alpha}}=F_{N} R_{e} \frac{\Psi}{W} \cos \left(\theta_{c}\right)-F_{T} \sin \left(\theta_{c}\right)
$$

$\mathrm{f}_{\beta}$ s'obtient de la même manière, ce qui donne au final :

$$
\left(\begin{array}{l}
f_{\alpha} \\
f_{\beta}
\end{array}\right)=\left(\begin{array}{c}
F_{N} R_{e} \frac{\Psi}{W} \cos \left(\theta_{c}\right)-F_{T} \sin \left(\theta_{c}\right) \\
F_{N} R_{e} \frac{\Psi}{W} \sin \left(\theta_{c}\right)+F_{T} \cos \left(\theta_{c}\right)
\end{array}\right)=\left(\begin{array}{cc}
\cos \left(\theta_{c}\right) & -\sin \left(\theta_{c}\right) \\
\sin \left(\theta_{c}\right) & \cos \left(\theta_{c}\right)
\end{array}\right)\left(\begin{array}{c}
F_{N} R_{e} \frac{\Psi}{W} \\
F_{T}
\end{array}\right)
$$

Dans le cas du régime permanent :

$$
\left(\begin{array}{c}
f_{\alpha} \\
f_{\beta}
\end{array}\right)=\left(\begin{array}{c}
-F_{N} R_{e} \frac{\Psi}{W} \cos (\omega t)+F_{T} \sin (\omega t) \\
-F_{N} R_{e} \frac{\Psi}{W} \sin (\omega t)-F_{T} \cos (\omega t)
\end{array}\right)=-\left(\begin{array}{cc}
\cos (\omega t) & -\sin (\omega t) \\
\sin (\omega t) & \cos (\omega t)
\end{array}\right)\left(\begin{array}{c}
F_{N} R_{e} \frac{\Psi}{W} \\
F_{T}
\end{array}\right)
$$

Si on note ces résultats en complexe, l'expression matricielle de l'équation mécanique : 


$$
\left(\begin{array}{cc}
m & 0 \\
0 & m
\end{array}\right)\left(\begin{array}{l}
\ddot{w}_{\alpha} \\
\ddot{w}_{\beta}
\end{array}\right)+\left(\begin{array}{cc}
c & 0 \\
0 & c
\end{array}\right)\left(\begin{array}{c}
\dot{w}_{\alpha} \\
\dot{w}_{\beta}
\end{array}\right)+\left(\begin{array}{cc}
k & 0 \\
0 & k
\end{array}\right)\left(\begin{array}{c}
w_{\alpha} \\
w_{\beta}
\end{array}\right)=\left(\begin{array}{ll}
n & 0 \\
0 & n
\end{array}\right)\left(\begin{array}{c}
v_{\alpha} \\
v_{\beta}
\end{array}\right)+\left(\begin{array}{c}
f_{\alpha} \\
f_{\beta}
\end{array}\right)
$$

devient :

$$
\left(j \omega m+c+\frac{k}{j \omega}\right) j \omega \underline{W}=n \underline{V}+\underline{F}
$$

avec :

$$
\underline{F}=-\left(\widetilde{F}_{N}+j F_{T}\right) \text { où } \widetilde{F}_{N}=F_{N} R_{e} \frac{\Psi}{W} \text { et } F_{T}=\mu \widetilde{F}_{N}
$$

Le diagramme vectorielle de la figure XXXX peut décrire géométriquement le comportement de moteur en régime permanent.

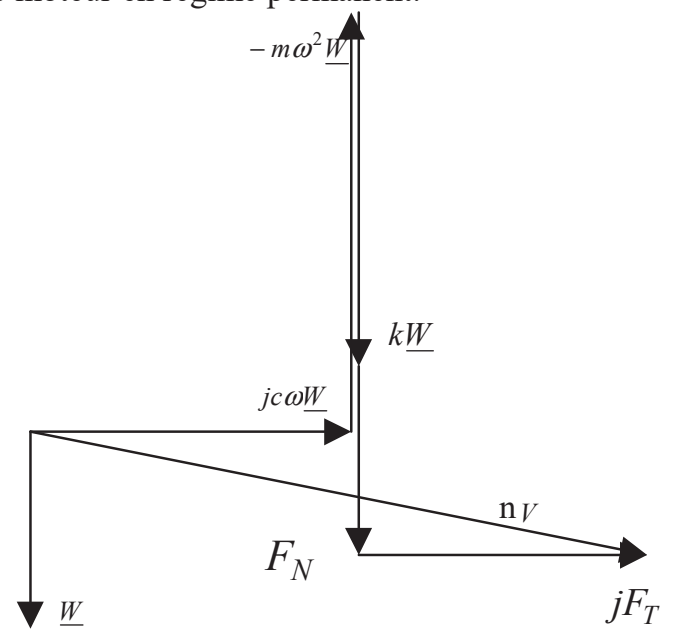

Figure 3. Diagramme vectoriel du moteur en régime permanent

\subsection{Expression des efforts dans le cas d'un contact réparti}

La raideur de contact n'est pas infinie et la zone de contact n'est pas en réalité ponctuelle. Le calcul des forces de réactions s'effectue de la même manière en intégrant la répartition d'effort développé à l'interface stator rotor. La largeur de contact $a$ et l'expression de la répartition de pression $p$ sont données par la théorie de Hertz dans le cas d'un contact cylindre/plan. L'onde progressive est approximée par un cylindre, le rotor par un plan. 

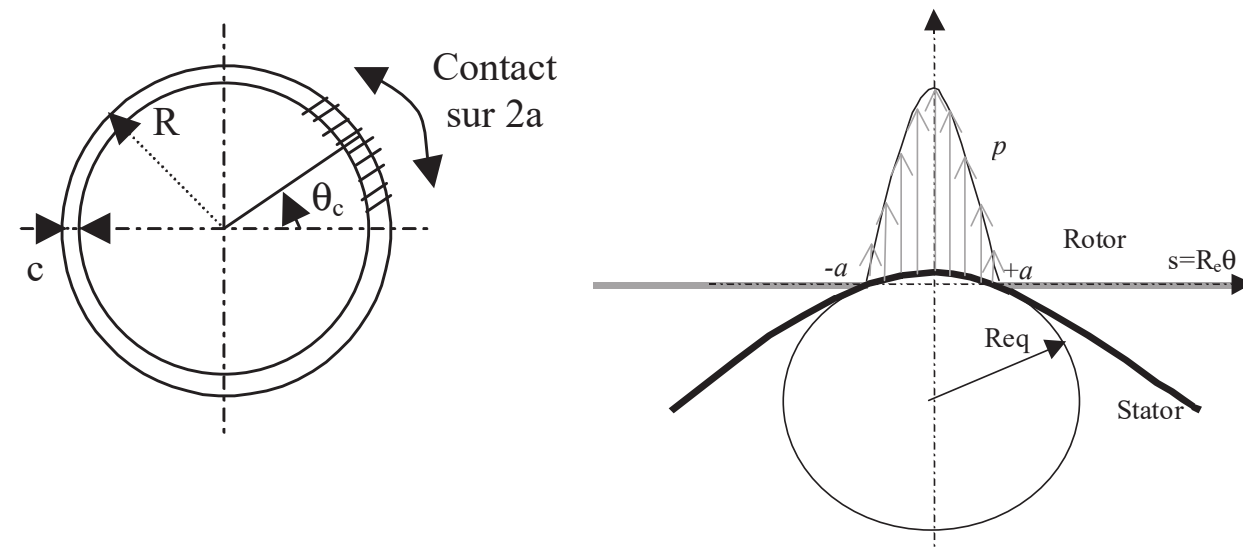

Figure 3. Contact Stator/Rotor

Le rayon du cylindre équivalent prend pour expression :

$$
R_{e q}=\frac{1}{\frac{\partial^{2} U_{x}}{\partial s^{2}}}=-\frac{R_{e}{ }^{2}}{U_{x}\left(\theta_{c}\right)}
$$

La largeur de contact prend alors comme valeur :

$$
a=2\left(\frac{L_{n} R_{e q}}{\pi E^{*}}\right)^{1 / 2} \text { ou } \theta_{a}=\frac{a}{R_{e}}=2\left(\frac{L_{n} R_{e q}}{\pi E^{*}}\right)^{1 / 2}
$$

avec :

- $L_{n}=\frac{F_{N}}{c}$, la charge linéique ;

- $\frac{1}{E^{*}}=\frac{1-\gamma_{\text {stator }}^{2}}{E_{\text {stator }}}+\frac{1-\gamma_{\text {rotor }}^{2}}{E_{\text {rotor }}}$, le module d'élasticité équivalent.

La répartition de pression $p$ est donnée par :

$$
p=P_{0}\left(1-\left(\frac{s}{a}\right)^{2}\right)^{1 / 2} \text { ou } p=P_{0}\left(1-\left(\frac{\theta-\theta_{c}}{\theta_{a}}\right)^{2}\right)^{1 / 2} \text { avec } P_{0}=\frac{2 L_{n}}{\pi a}
$$

C'est la partie tangentielle $U_{\theta}$ du mouvement elliptique qui fournit la vitesse au rotor. Son expression est dans le référentiel défini figure XXX ou $s$ est la position des point autour du contact : 


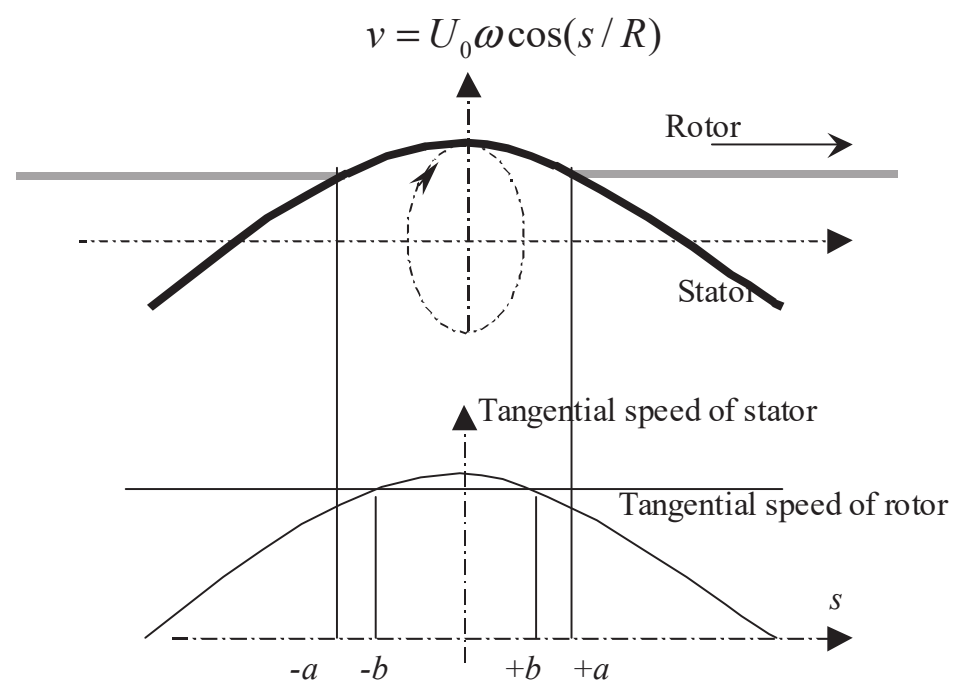

Figure 3. Profil des vitesses tangentielles au niveau du contact

Sur la figure XXXX des vitesses tangentielles vibratoires, l'abscisse $b$ correspond au point pour lequel vitesse tangentielle du stator est égale à la vitesse tangentielle $V_{r}$ du rotor. On a :

$$
V_{r}=U_{0} \omega \cos (b / R) \text { et } b=R \cdot \operatorname{ar} \cos \left(V_{r} / U_{0} \omega\right)
$$

L'effort fournit au rotor dépend de la vitesse relative :

- Pour $v>V_{r}$ ou $-b<s<b$ : moteur ;

- Pour $v<V_{r}$ ou $-a<s<-b$ ou $b<x<a$ : frein.

En prenant une loi de frottement sec de type $d F=\mu \cdot p \cdot s g\left(v-V_{r}\right) d S$ ( $\mu$ coefficient de Coulomb), le couple se calcule de la façon suivante :

$$
\begin{gathered}
C=2 \mu R\left(\int_{0}^{b} p(s) c d s-\int_{b}^{a} p(s) c d s\right)=2 \mu c R P_{0}\left(\int_{0}^{b} \sqrt{1-(s / a)^{2}} d s-\int_{b}^{a} \sqrt{1-(s / a)^{2}} d s\right) \\
C=2 \mu a c R P_{0}\left(\arcsin \left(\frac{b}{a}\right)+\frac{b}{a} \sqrt{1-\left(\frac{b}{a}\right)^{2}}-\frac{\pi}{4}\right)
\end{gathered}
$$

Et pour des vitesses faibles ( $b \geq a$ ) il prend la valeur maximale :

$$
C_{\text {max }}=\frac{\pi}{2} \mu a c R P_{0}=\mu F_{\text {pres }} R
$$

A l'aide de ces expressions, la caractéristique courbe/vitesse prend pour la forme représentée figure XXX. 


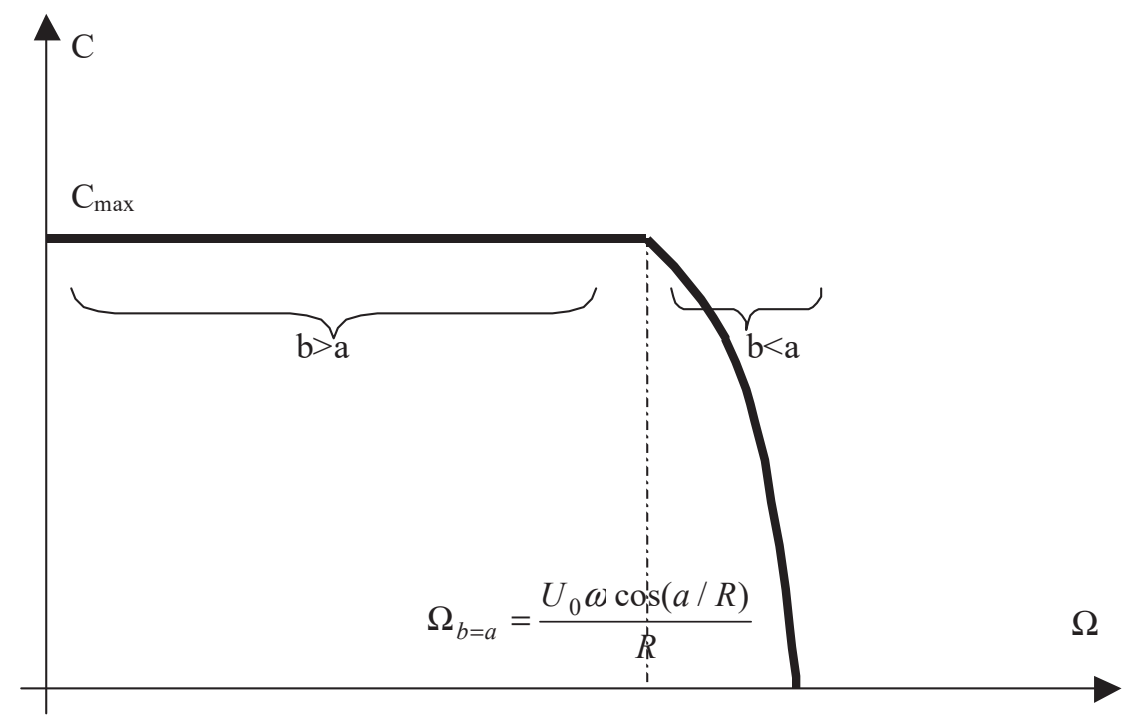

Figure 3. Caractéristique couple/vitesse

Les forces de réactions modales s'obtiennent comme pour le cas du contact ponctuel, mais en intégrant la projection de l'effort répartie sur la zone de contact :

- Pour la pression normale :

$$
\begin{gathered}
\widetilde{F}_{N}=-\int c P(\theta) \frac{\partial U \gamma(\theta)}{\partial w_{\alpha}} R_{e} d \theta=\int_{\theta_{c}-\theta_{a}}^{\theta_{c}+\theta_{a}} c P(\theta) R_{e} \frac{\Psi}{W} \cos (\theta) R_{e} d \theta \\
\widetilde{F}_{N}=c R_{e}^{2} \frac{\Psi}{W} P_{0} \frac{\pi \theta_{a}}{(\pi / 2)^{2}-\theta_{a}^{2}} \cos \left(\theta_{a}\right) \sin \left(\theta_{c}\right)
\end{gathered}
$$

- $\quad$ Pour le frottement tangentiel :

$$
\begin{gathered}
F_{T}=\int c \mu P(\theta) \frac{\partial U \gamma(\theta)}{\partial w_{\alpha}} R_{e} d \theta=-\int_{\theta_{c}-\theta_{a}}^{\theta_{c}+\theta_{a}} c \mu P(\theta) \sin (\theta) R_{e} d \theta \\
F_{T}=c \mu P_{0} R_{e} \frac{\pi \theta_{a}}{(\pi / 2)^{2}-\theta_{a}^{2}} \cos \left(\theta_{a}\right) \cos \left(\theta_{c}\right)
\end{gathered}
$$

- $\quad$ Pour le frottement radial :

$$
F_{R}=c \mu P_{0} R_{e} \frac{2 \theta_{a}}{(\pi / 2)^{2}-\theta_{a}^{2}}\left(\sin \left(\theta_{a}\right) \frac{\pi}{2}-\theta_{a}\right) \cos \left(\theta_{c}\right)
$$

Les forces modales s'exprime comme précédemment avec l'expression :

$$
\left(\begin{array}{l}
f_{\alpha} \\
f_{\beta}
\end{array}\right)=\left(\begin{array}{cc}
\cos \left(\theta_{c}\right) & -\sin \left(\theta_{c}\right) \\
\sin \left(\theta_{c}\right) & \cos \left(\theta_{c}\right)
\end{array}\right)\left(\begin{array}{c}
F_{N} \\
F_{T}+F_{R}
\end{array}\right)
$$


RIGE - proposition d'article

L'expression de l'équation mécanique en représentation complexe traitée précédemment dans le cas du contact ponctuel reste valable ainsi que sa représentation vectorielle. Seules changent les expressions des efforts traduisant le contact stator/rotor.

\subsection{Application numérique}

Pour un moteur à rotation de mode assimilé un cylindre (150 $\mathrm{mm}$ de long, diamètre extérieur de $50 \mathrm{~mm}$, intérieur $20 \mathrm{~mm}$ ), avec six céramiques P1 189 de $1 \mathrm{~mm}$ d'épaisseur par phase, les résultats et le schéma équivalents de la figure XXX sont obtenus avec un effort normal de $1000 \mathrm{~N}$ et des vibrations $w$ de $4 \mu \mathrm{m}$ :

- Paramètres électromécaniques : $\mathrm{M}=650 \mathrm{~g}, \mathrm{~K}=7,28.10^{8}, \mathrm{f}=5.3 \mathrm{kHz}, \mathrm{Q}_{\mathrm{m}}=200$, $\mathrm{N}=0,165 \mathrm{~N} / \mathrm{V}$;

- Caractéristiques mécaniques couple et vitesse : $\mathrm{C}_{\max }=6,25 \mathrm{~N} . \mathrm{m}, \Omega_{\mathrm{lim}}=48 \mathrm{tr} / \mathrm{min}$;

- Tension d'alimentation : $\mathrm{V}_{\mathrm{alim}}=275 \mathrm{~V}$.

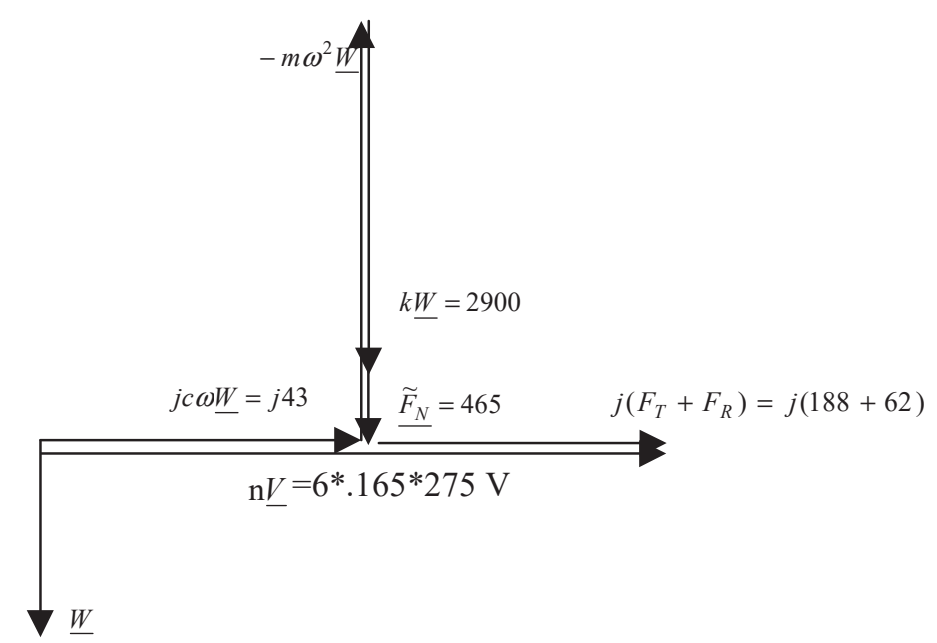

Figure 3. Diagramme vectoriel du moteur en régime permanent 


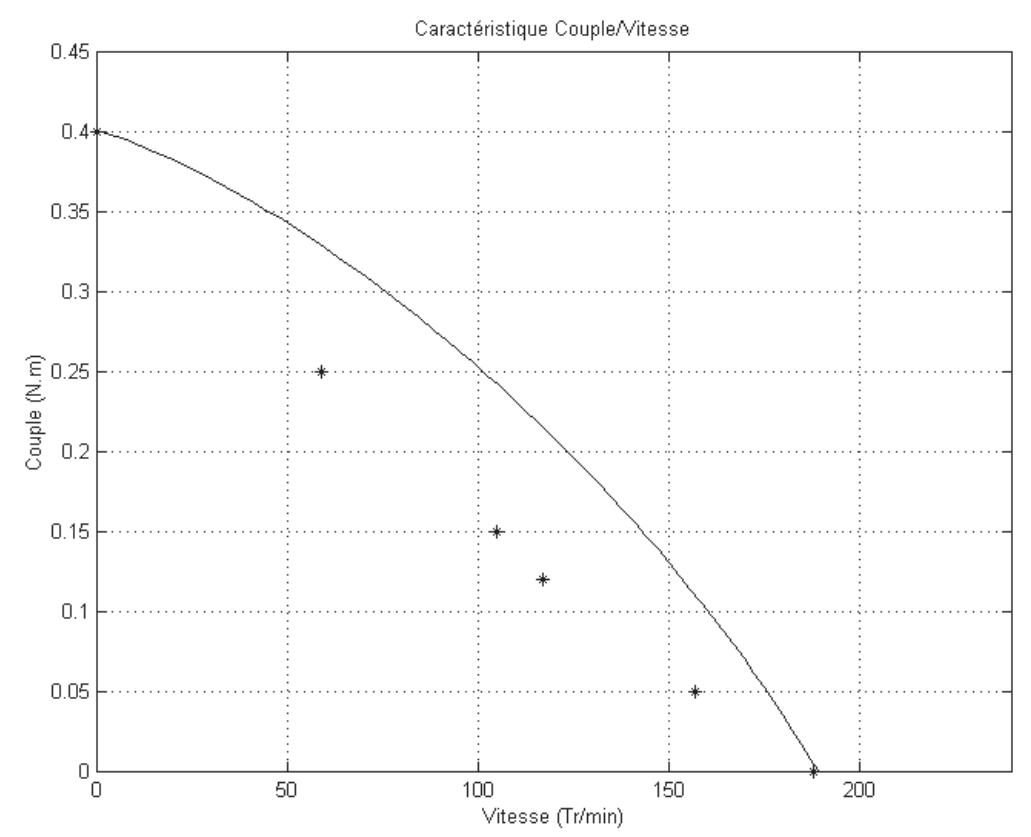

\section{Conclusion}

L'article présente un modèle global simple du moteur à rotation de mode en régime permanent. La méthode d'étude passe, comme la conversion d'énergie dans les moteurs piézo-électriques, par deux étapes successives. La première concerne la conversion électromécanique de l'énergie électrique appliquée sur les phases en énergie vibratoire. Elle est caractérisée par différents paramètres tels que le facteur d'effort, la masse et la raideur modale autour de la fréquence de résonance utilisée. Ces paramètres peuvent être calculés de manière analytique ou numérique. Le calcul analytique se base sur la théorie des poutres pour trouver la fréquence de résonance du mode considéré ainsi qu'une approximation de la déformée du stator. Cette dernière est intégrée dans un Lagrangien afin d'en déduire les valeurs des paramètres précédemment cités. De la même manière, les éléments finis permettent d'obtenir les grandeurs énergétiques caractéristiques du Lagrangien (énergie potentielle élastique et énergie cinétique) ainsi le couplage électromécanique. Ces calculs, analytiques et numériques, ont été illustrés sur deux exemples simples : un transducteur Langevin et un stator de moteur à rotation de mode. Une bonne corrélation des résultats entre les deux méthodes est observée. La deuxième conversion, mécano-mécanique, est prise en compte de manière purement analytique. Les forces de frottement, déduites de la théorie de Hertz, sont intégrées au Lagrangien sous la forme de pseudopotentiels. L'ensemble se ramène à un schéma vectoriel équivalent extrêmement 
RIGE - proposition d'article

simple qui rend compte: du phénomène de résonance et du transfert électromécanique d'énergie. On peut rapprocher ce schéma des schémas équivalents de machines rencontrés en électrotechnique classique. Cet outil est utile au concepteur de machine dans les étapes de dimensionnement et peut être appliqué à d'autres types de moteurs que le rotation de mode. Les grandeurs caractéristiques déduites ici trouvent également un intérêt pour la représentation du moteur en régime dynamique et la mise en place d'une commande appropriée [BUD 02].

\section{Bibliographie}

[BAO 00] Bao X., Bar-Cohen Y., « Complete modeling of rotary ultrasonic motors actuated by traveling flexural waves », Proceedings of SPIE's $7^{\text {th }}$ Annual International Symposium on Smart Strcutures and Materials, 1-5 march, 2000, Newport, CA.

[CAN ] Canon, EF Lens Work II, plaquette de présentation des optiques Canon.

[CAN ] Canon, Vibration driven motor, brevet CA2048400.

[GER] Geradin G., Rixen D., Théorie des vibrations (application à la dynamique des structures), Masson.

[IKE ] Ikeda T., Fundamentals of Piezoelectricity, Oxford Science Publications.

[KUR 89] Kurosawa M. et al. , An ultrasonic motor using bending vibrations of a short cylinder, IEEE Transactions on ultrasonics ferroelectrics and frequency control, vol. 36, $\mathrm{n}^{\circ}$ 5, sept. 1989.

[MIN 96] Minotti P. et al., Moteur piezo-électrique à onde progressive : I. modélisation de la conversion d'énergie mécanique à l'interface stator/rotor, Journal de Physique III., 1996, vol. 6, n¹0, p. 1315-1337

[MOR 96] Morita T. et al., Design of a cylindrical ultrasonic micromotor to obtain mechanical output, Jpn. J. Appl. Phys., Vol. 35 (1996), pp. 3251-3254

[MOR 99] Morita T. et al., Cylindrical micro ultrasonic motor utilizing bulk lead zirconate titanate (PZT), Jpn. J. Appl. Phys., Vol. 38 (1999), pp. 33473350

[NOG] Nogarede B., Machines tournantes : conversion électromécanique de l'énergie, Technique de l'Ingénieur, D3410

[PIN 01] Pin Lu et al., A kinematic analysis of cylindrical ultrasonic micromotors, Sensors and Actuators A 87 (2001) 194-197

[ROU 95] Rouchon J.F., Kapsa Ph., The elastic contact area between a sinusoidal indentor and a layered solid : application to calculation of ultrasonic motors performances, International Tribology Conference, oct. Nov. 1995, Yokohama, Japan. 
[SAS] Sashida T., Kenjo T., An introduction to ultrasonic motors, Clarendon Press, OXFORD, 1993.

[TIM ]

Timoshenko S., Résistance des matériaux, Dunod.

$[\mathrm{UEH}]$ Ueha S., Tomikawa Y., Ultrasonic Motors, Oxford Science Publication, 1993

[ZHO 00] Zhou G., The performance and design of ultrasonic vibration system for flexural mode, Ultrasonics 38 (2000), Elsevier Science, pp. 979-984

[BUD 02] Budinger M., Giraud F., Rouchon J-F, Lemaire-Semail B., Nogarede B., Feeding and control electronic of a piezoelectric actuator, ACTUATOR'2002, juin 2002, Bremen, Allemagne 